\title{
A minimally invasive catheter-based ultrasound technology for therapeutic interventions in brain: initial preclinical studies
}

\author{
Goutam Ghoshal, PhD, ${ }^{1}$ Lucy Gee, PhD, ${ }^{2,3}$ Tamas Heffter, MSEE, ${ }^{1}$ Emery Williams, BS, ${ }^{1}$ \\ Corinne Bromfield, DVM, ${ }^{4}$ Laurie Rund, PhD, ${ }^{4}$ John M. Ehrhardt, DVM, ${ }^{4}$ Chris J. Diederich, PhD, ${ }^{5}$ \\ Gregory S. Fischer, PhD, ${ }^{6}$ Julie G. Pilitsis, MD, PhD, ${ }^{2,3}$ and E. Clif Burdette, $\mathrm{PhD}{ }^{1}$
}

\begin{abstract}
${ }^{1}$ Acoustic MedSystems, Inc., Savoy, Illinois; Departments of ${ }^{2}$ Neuroscience and Experimental Therapeutics and ${ }^{3}$ Neurosurgery, Albany Medical Center, Albany, New York; ${ }^{4}$ Department of Animal Sciences, University of Illinois, Urbana, Illinois; ${ }^{5}$ Department of Radiation Oncology, University of California, San Francisco, California; and ${ }^{6}$ Department of Mechanical Engineering, Worcester Polytechnic Institute, Worcester, Massachusetts
\end{abstract}

OBJECTIVE Minimally invasive procedures may allow surgeons to avoid conventional open surgical procedures for certain neurological disorders. This paper describes the iterative process for development of a catheter-based ultrasound thermal therapy applicator.

METHODS Using an ultrasound applicator with an array of longitudinally stacked and angularly sectored tubular transducers within a catheter, the authors conducted experimental studies in porcine liver, in vivo and ex vivo, in order to characterize the device performance and lesion patterns. In addition, they applied the technique in a rodent model of Parkinson's disease to investigate the feasibility of its application in brain.

RESULTS Thermal lesions with multiple shapes and sizes were readily achieved in porcine liver. The feasibility of catheter-based focused ultrasound in the treatment of brain conditions was demonstrated in a rodent model of Parkinson's disease.

CONCLUSIONS The authors show proof of principle of a catheter-based ultrasound system that can create lesions with concurrent thermode-based measurements.

https://thejns.org/doi/abs/10.3171/2017.11.FOCUS17631

KEY WORDS HIFU; focused ultrasound; ablation; Parkinson's disease

$\mathrm{T}$ HERMAL ablation and hyperthermia are important treatment options for soft tissue and brain diseases. ${ }^{1}$ Hyperthermia can be directly cytotoxic or may be used as an adjunct to radiation and chemotherapy; a Phase III trial demonstrated a survival advantage for combining interstitial hyperthermia with interstitial brachytherapy treatment for glioblastoma multiforme. ${ }^{17}$ Localized thermal ablation causes protein denaturation, necrosis, and coagulation in the treatment region, allowing the treatment to kill unhealthy cells while sparing healthy tissue away from the targeted tissue. Over the last several years, transcranial MR-guided focused ultrasound (tcMRgFUS) has gained traction as an alternative treatment for brain disorders. ${ }^{3,11}$ Short-term results with tcMRgFUS are exciting, with reduction of hand tremor by $75 \%$ and overall tremor by $56 \%$ in 15 essential tremor patients. ${ }^{3}$ Immediately after treatment, 4 patients reported persistent paresthesias, which subsequently resolved in all cases but one. Five patients had unsteadiness and objective decline in gait, but in all 5 cases, these symptoms had resolved at 1 month. Long-term follow-up is needed to understand whether high-intensity focused ultrasound (HIFU) has the longevity of deep brain stimulation (DBS) and thalamotomy (75\%-84\% at $4-5$ years). ${ }^{7,14,15}$ Additional insight through

ABBREVIATIONS DBS = deep brain stimulation; HIFU = high-intensity focused ultrasound; LAT = limb use asymmetry test; OFT = open field test; PD = Parkinson's disease; RFA = radiofrequency ablation; STN = subthalamic nucleus; tcMRgFUS = transcranial MR-guided focused ultrasound; TTC = triphenyltetrazolium chloride; $6-\mathrm{OHDA}=$ 6-hydroxydopamine. 
further study is also needed to determine which dose results in optimal long-term tremor control. Transcranial MRgFUS differs from other ablative procedures and DBS in that it is noninvasive and delivered externally. A major barrier to its use remains the capital investment required for the initial acquisition of the system. Further, the size of lesions is limited, as is the ability to target regions near the skull. In this study, we explored catheter-based options that could be used in an intraoperative MR setting or with thermometry sensing coupled to the catheter.

\section{Methods}

\section{Porcine Liver Studies}

The swine studies were approved by the University of Illinois, Urbana, institutional animal care and use committee. First, an ex vivo test was performed in porcine liver tissue taken from a female farm pig. The ex vivo test used a TheraVision ultrasound ablation system with Acoustx applicators (Acoustic MedSystems, Inc.) to estimate lesion size and shape (Fig. 1). ${ }^{6}$ Then, an in vivo study was performed in a female farm pig $(90 \mathrm{~kg})$ under general anesthesia. Specifically, we visualized the liver using diagnostic ultrasound and inserted the catheter ultrasound applicator using previously described techniques. ${ }^{9}$ A fine thermode couple was inserted within $2-3 \mathrm{~mm}$ of the catheter probe to estimate temperature during treatment. Following lesion creation, the pig was immediately euthanized by intravenous administration of sodium pentobarbital in accordance with American Veterinary Medical Association guidelines, and its liver was subsequently removed for histopathological examination. Specifically, gross assessment for ablation was performed and liver tissue was then stained with triphenyltetrazolium chloride (TTC), a tissue viability stain, which made the treated region and normal tissue appear in different colors, to allow for easy measurement of the ablation zone. These experiments were geared at determining proof of principle of liver ablation and at correlating thermode measurements of energy delivery with predicted ablation volumes.

\section{Rodent Survival Surgeries}

All rodent use was approved by the institutional animal care and use committee at Albany Medical College. Experiments were performed on 7-week-old male Sprague Dawley rats that weighed 200-350 g (obtained from Charles River); 2 naive and 5 parkinsonian rodents were used.

For the parkinsonian cohort, general anesthesia was induced using 2\% isoflurane via an inhalant system (Harvard Apparatus). The rats' body temperature was maintained at $37^{\circ} \mathrm{C}$ with a warming blanket. They were injected intraperitoneally with desipramine hydrochloride $(25 \mathrm{mg} /$ $\mathrm{kg})$ and pargyline $(50 \mathrm{mg} / \mathrm{kg}) 30$ minutes prior to 6-hydroxydopamine (6-OHDA) injection to increase 6-OHDA efficacy. Animals were shaved and then placed into the stereotactic frame (David Kopf Instruments). The area was sterilized with povidone-iodine and the incision site was injected with $2 \%$ xylocaine. A burr hole was drilled in the skull over the right medial forebrain bundle at $4.4 \mathrm{~mm}$ posterior and $1.5 \mathrm{~mm}$ lateral to the bregma. An injection
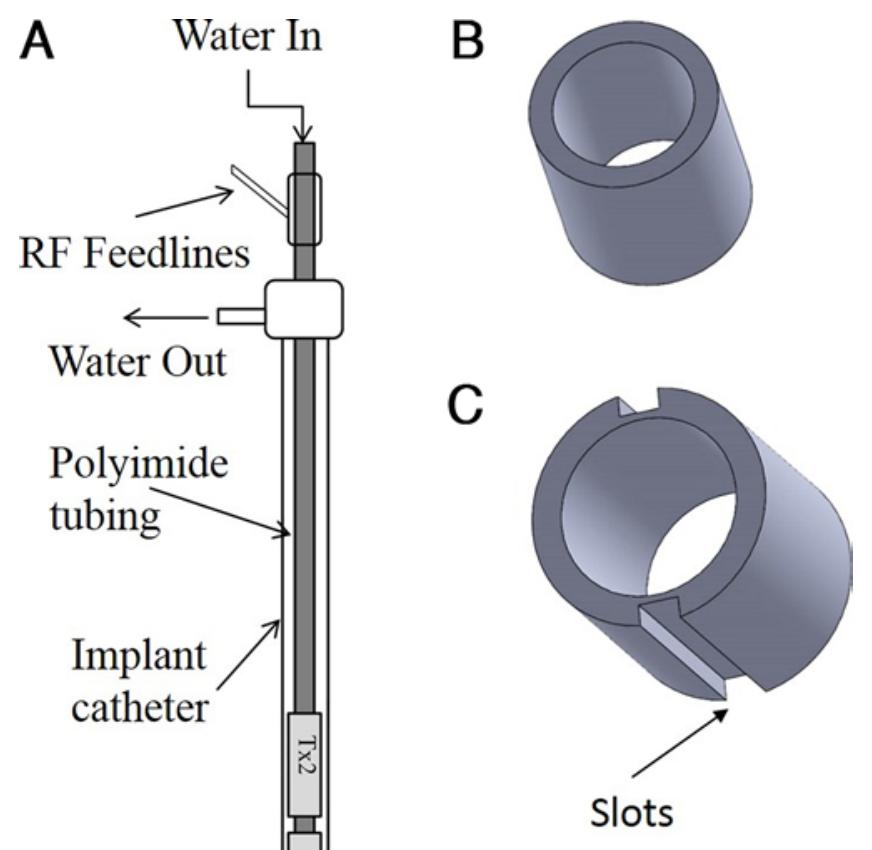

FIG. 1. A: Diagrammatic representation of a high-intensity therapeutic ultrasound interstitial catheter. B and C: Cross-sectional views of a $360^{\circ}$ tubular transducer $(\mathrm{B})$ and $\mathrm{a} 180^{\circ}$ tubular transducer $(\mathrm{C})$. $\mathrm{RF}=$ radiofrequency.

needle (10- $\mu 1$ Hamilton syringe) was lowered $7.8 \mathrm{~mm}$ ventral from the dura, and $4.5 \mu \mathrm{l}$ of 6-OHDA ( $3 \mu \mathrm{g} / \mu \mathrm{l}$, made up with $0.1 \%$ ascorbic acid, Sigma-Aldrich) was injected at a rate of $0.5 \mu \mathrm{l} /$ minute. The needle was left in place for an additional 10 minutes after 6-OHDA injection. The animals were allowed to recover for 2 weeks. After 2 weeks of recovery, Parkinson's disease (PD) phenotype was confirmed by means of the limb-use asymmetry test (LAT). ${ }^{18}$ Additionally, animals underwent assessment with a step test, open field test (OFT), and rotarod test as markers of mobility and behavior. Catheter-based ultrasound therapy was then performed the following day.

According to a similar protocol as above, another burr hole was made $4.1 \mathrm{~mm}$ posterior and $2.5 \mathrm{~mm}$ lateral to the bregma. The ultrasound catheter was inserted $7.6 \mathrm{~mm}$ ventral from the dura to the right subthalamic nucleus (STN). The 2 naïve animals and 2 of the PD animals were treated for 3 minutes with $5 \mathrm{~W}$ of acoustic power, and the other $3 \mathrm{PD}$ animals were treated for 3 minutes with $3 \mathrm{~W}$ of acoustic power. Antibiotic cream (Neosporin) was then applied around the surgical site, and penicillin $(80 \mu \mathrm{g} / \mathrm{kg})$ was subcutaneously injected to prevent infection.

Behavioral testing, including the LAT, step test, OFT, and rotarod test, was performed at 24 hours, 72 hours, and 5 days after ultrasound treatment. Animals were killed 24 hours (naïve rats) or 5 days (PD rats) after ultrasound treatment (or earlier if in extremis). Specifically, they underwent transcardiac perfusion with $60 \mathrm{ml}$ heparin followed by $60 \mathrm{ml} \mathrm{4 \%}$ paraformaldehyde. The brains were removed and post-fixed for 24 hours with $4 \%$ paraformal- 


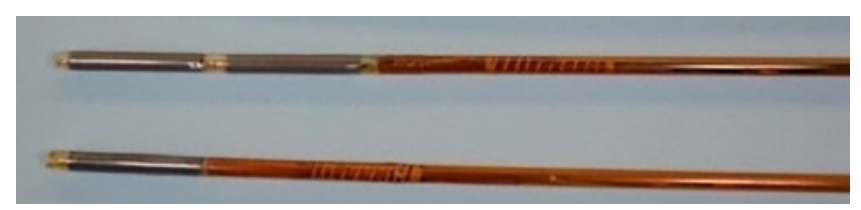

FIG. 2. Fabricated applicator with single (bottom) and double (top) transducer elements.

dehyde and then incubated for $48-72$ hours in $30 \%$ sucrose at $4^{\circ} \mathrm{C}$. With a cryostat, brains were frozen before being sliced to obtain $60-\mu \mathrm{m}$-thick sections. Sliced sections were stored for $24-48$ hours prior to staining at $4^{\circ} \mathrm{C}$. The sections were stained with cresyl violet and examined to confirm lesion location and lesion size, as described by Gee et al. ${ }^{5}$

\section{Data Analysis}

Statistical analyses were performed with GraphPad Prism (GraphPad Software, Inc.) using parametric tests for data sets with normal distributions. Data were represented with the mean \pm standard error of the mean (SEM) for histological studies or standard deviation (SD) for behavioral studies; $\mathrm{p}<0.05$ was accepted as significant.

\section{Catheter-Based Ultrasound Applicators}

The catheter-based ultrasound interstitial applicators used in these studies consisted of multi-element tubular piezoelectric transducers, designed to ensure MR compatibility, although MR was not used in these experiments
(Figs. 1 and 2). ${ }^{8,10,13}$ They are $7-15 \mathrm{~mm}$ long and have a 1.5-mm diameter and are mounted on a hollow polyimide tube. The applicator is maintained in a flexi-needle catheter to allow for circulation of degassed water to cool the transducers during treatment (Fig. 3A) and to provide effective acoustic coupling to the target tissue. ${ }^{16}$ To maximize output of thermal energy, the frequency with greatest acoustic output and efficiency was used. Typically, acoustic efficiency ranged from $50 \%$ to $65 \%$ with operating frequencies between 6.5 and $7.5 \mathrm{MHz}$.

\section{Results}

\section{Porcine Studies}

A 13-gauge cannula was used to guide the treatment applicator to the target. We show data from transducers with $360^{\circ}$ active zones (Fig. $1 \mathrm{~B}$ and C). A $360^{\circ}$ active zone creates a cylinder of ablation, while a $180^{\circ}$ zone creates half that volume (Fig. 3) with a semicylindrical pattern, and a $90^{\circ}$ active zone results in a sector-shaped energy pattern., ${ }^{2,12}$ The efficiency and resonant frequency of each transducer was calculated using measurements of impedance and radiation force balance. The ex vivo study revealed that a lesion with a diameter of $3 \mathrm{~cm}$ and depth of $1.5 \mathrm{~cm}$ was induced with an acoustic power of $7 \mathrm{~W}$ and exposure time of 6 minutes (Fig. 4). Next, in vivo, to create a lesion with a diameter of $2.4 \mathrm{~cm}$ and depth of $2 \mathrm{~cm}$, an acoustic power of $8 \mathrm{~W}$ for 8 minutes was employed (Fig. 5). More power density was needed for the in vivo experiment to overcome blood perfusion. Cylindrical lesions were created by using the probe with a $360^{\circ}$ pattern,

\section{A Acoustx US Catheter}
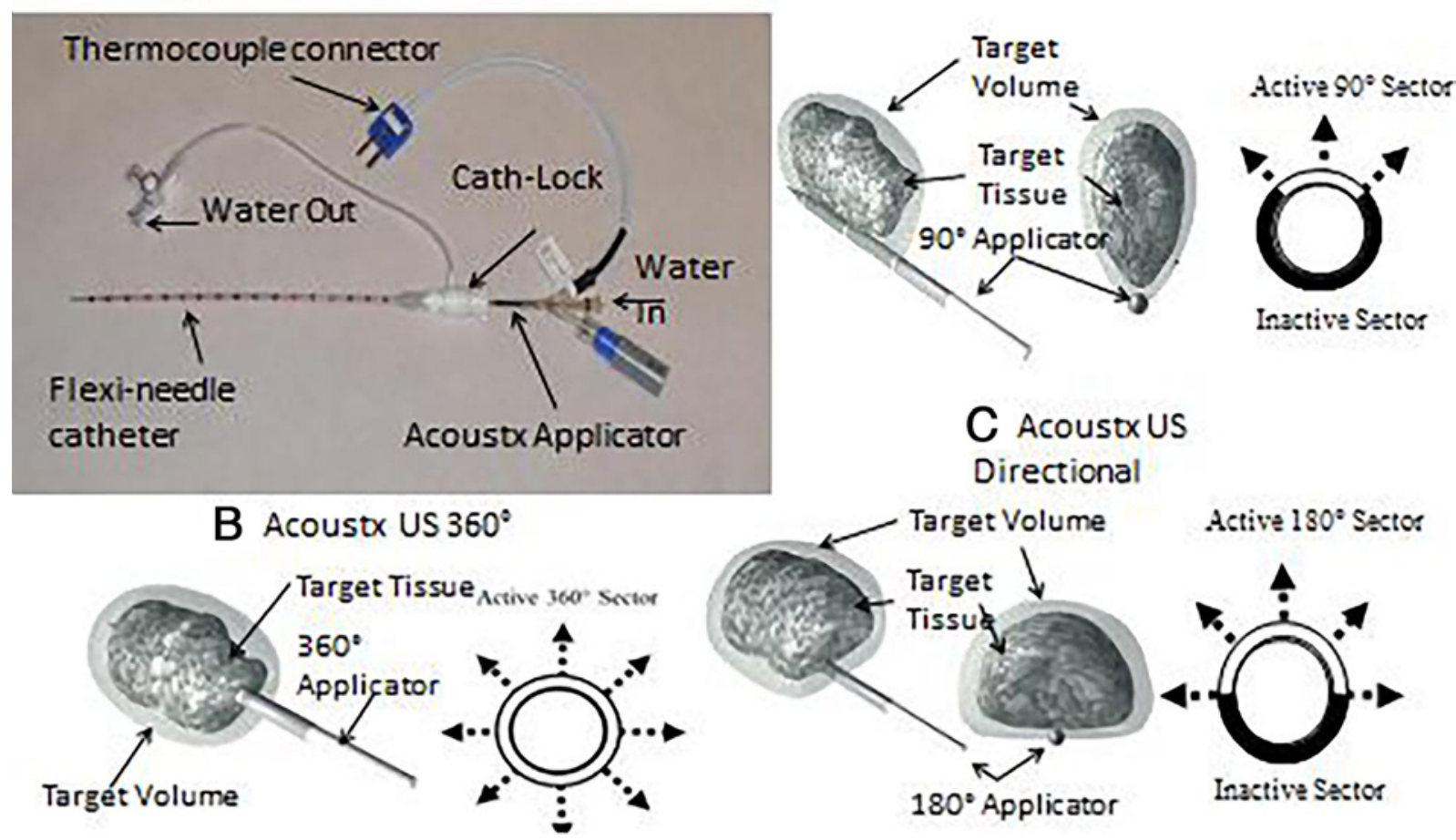

FIG. 3. A: Catheter-based ultrasound applicator for percutaneous conformal thermal ablation to targets in soft tissues. B: $360^{\circ}$ pattern. C: $90^{\circ}$ and $180^{\circ}$ patterns. Cath = catheter; US = ultrasound. 

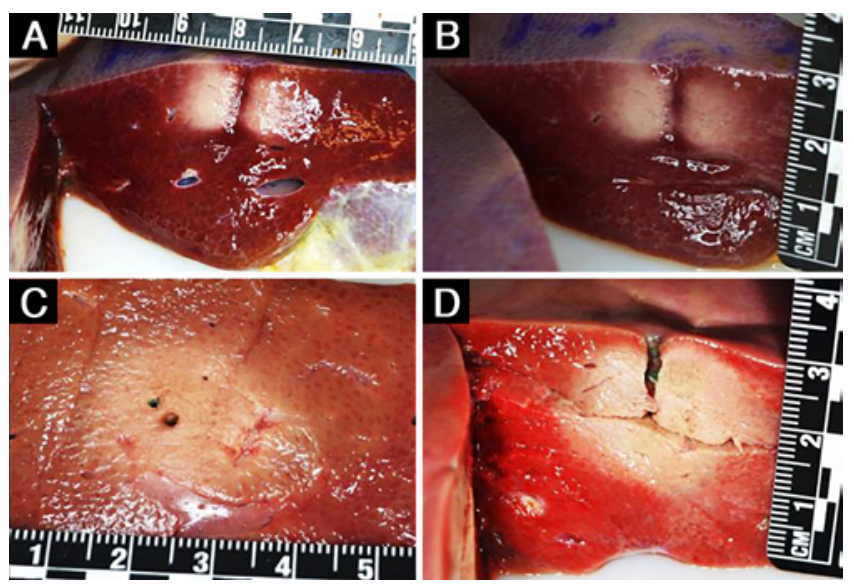

FIG. 4. Representative gross pathology images of the ablated pig liver tissue obtained with the single transducer element ( $A$ and $\mathbf{B}$ ) and 2-element ( $\mathbf{C}$ and $\mathbf{D}$ ) configurations, each with a single insertion of the Acoustx applicator. The rulers are marked in centimeters. The image in D was obtained after TTC staining.

and a $190^{\circ}$ angular lesion pattern was produced by probes sectored with a $180^{\circ}$ pattern.

\section{Rodent Studies}

In our survival studies in PD rodents, we opted to start with an acoustic power of $5 \mathrm{~W}$ for 3 minutes to account for the smaller size of the rodent brain. Ultrasound $a b-$ lation was performed in the 2 naïve animals as proof of principle, and these animals had no notable neurological deficit 24 hours later, when they were killed. In the first 2 of the 6-OHDA rats that were treated with ultrasound, the same dose was employed, but neither survived the therapy. Thus for the final $3 \mathrm{PD}$ rats the dose was reduced to $3 \mathrm{~W}$ for 3 minutes. No significant changes in parkinsonian symptoms, gait, locomotion, or signs of anxiety were noted from presurgical testing to 5 days following treatment (Fig. 6). Prior to treatment, LAT scores were considered parkinsonian, as the animals exhibited a greater than $80 \%$ bias toward their right paw (mean \pm SD $90.59 \%$ $\pm 2.51 \%$ ); however, only a subset of treated animals explored the cylinder enough to provide an accurate score on LAT (Fig. 6A). We used the step test as a surrogate marker and showed a decrease from $70.60 \% \pm 14.71 \%$ to a range of $48.33 \%-67.74 \%$ at 5 days (Fig. 6B). In the OFT (n $=3$ ), there was no significant change in distance traveled

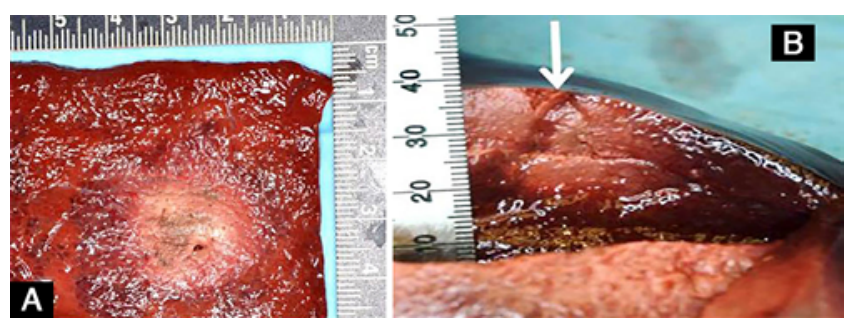

FIG. 5. Representative gross pathology images of the ablated pig (A and B) liver tissue obtained after ablation using the Acoustx applicator with 2 elements. The arrow in B indicates the insertion direction of the applicator. The tape in B is marked in millimeters.
A Limb-use Asymmetry Test $\mathbf{B}$
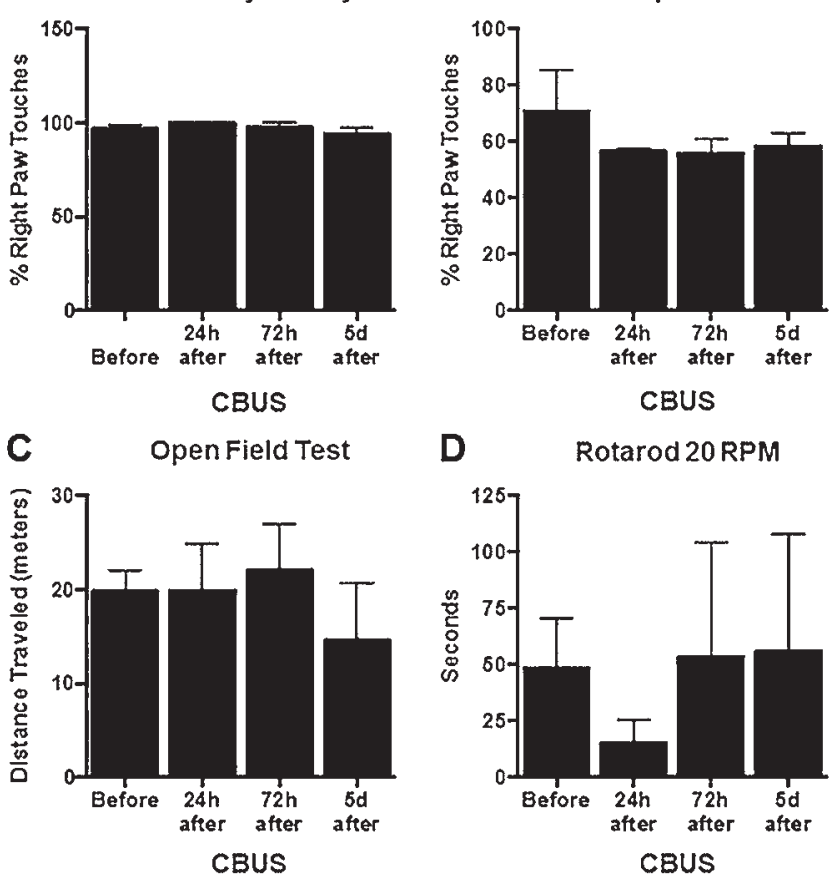

FIG. 6. Results of behavioral testing of PD rats $(n=3)$ pre- and posttreatment. No significant changes in limb asymmetry were noted following treatment on either the LAT $(\mathbf{A})$ or step test $(\mathbf{B})$. Locomotion and gait were also unaffected $(\boldsymbol{C}$ and $\mathbf{D}) ; p>0.05$. CBUS = catheter-based ultrasound; $d=$ days; $h=$ hours.

(Fig. 6C), indicating that the animals did not acquire a significant locomotor deficit after treatment. Furthermore, animals entered the center and spent a similar amount of time in the center, indicating that this procedure did not increase their anxiety level (data not shown). On the rotarod, animals averaged $48.33 \pm 22.44$ seconds prior to application and returned to similar values at 72 hours (53.33 \pm 50.85 seconds, $n=3$ ) (Fig. 6D). Histological analysis demonstrated the lesion in the right STN of a naïve animal. Animals treated with 3 minutes of HIFU at $3 \mathrm{~W}$ did not show any edema, and the mean $( \pm$ SEM) lesion size was calculated as $2.01 \times 10^{5} \pm 5.11 \times 10^{4} \mathrm{~mm}^{3}$ (Fig. 7B).

\section{Discussion}

Here we demonstrate proof of principle of catheterbased ultrasound ablation in porcine liver ex vivo, porcine liver in vivo, and rat brain. The porcine data afforded us the opportunity to determine which acoustic power correlates with lesion size. Based on histological results, the appropriate duration and wattage using these catheters in rodent brain appears to be $3 \mathrm{~W}$ for 3 minutes. Future work will focus on determining the actual amount of thermal energy being administered during that exposure. Additionally, the applicator diameter used in the rodent work described herein was $2.2 \mathrm{~mm}$; future work will use a 0.5 -mm-diameter applicator to mimic the size of existing DBS electrodes used in rodents (Fig. 7C).

Invasive radiofrequency ablation (RFA) has been used previously to perform thalamotomy/pallidotomy with 


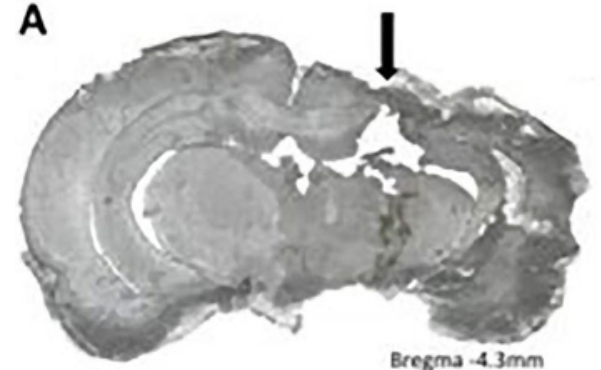

applicator placement

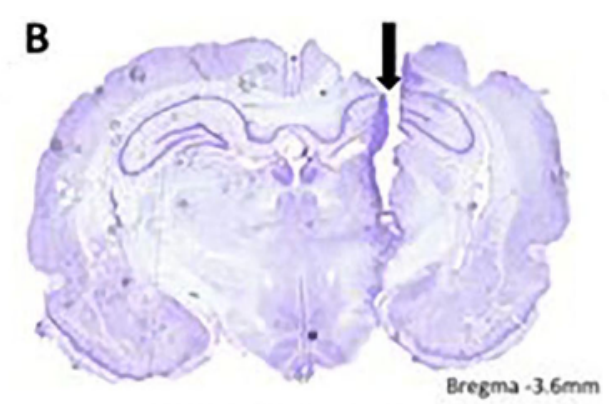

$3 W 3$ minutes

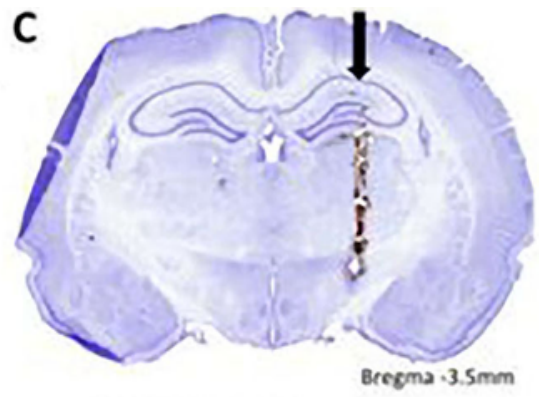

STN DBS electrode

FIG. 7. Histological sections of rodent brains. A: Unstained section showing catheter position in the brain of a naïve rat. B: Cresylviolet-stained section of a brain treated at $3 \mathrm{~W}$ for 3 minutes. C: Cresyl-violet-stained section of the brain of a PD rodent treated with STN DBS (provided for purposes of comparison). Black arrows designate track for catheter or electrode in each brain slice.

long-standing clinical benefit. ${ }^{7}$ However, complications were noted in $9 \%-23 \%$ of patients. ${ }^{4}$ RFA probes operate by thermal conduction to create spherical/ellipsoid treatment regions. The size of the ablation zone is limited by probe size and overheating of immediately adjacent tissue resulting in charring adjacent to the electrodes, effectively producing thermal insulation rather than conduction, without any control of the directionality of the ablation zone. The development of a novel energy-based technology that effectively penetrates independently of conduction and creates spatially conformal control of the ablation zone would constitute a significant improvement in lesioning.

Catheter-based ultrasound therapeutic devices are being developed for thermal treatment of brain tissue. Current technology includes 1- to 2.2-mm-diameter probes that can be inserted under stereotactic guidance into brain tissue through a burr hole. This device would enable the physician to perform a "directional ablation" of variable intensity distributions with greater control of the ablation zone shape, size, and volume, both laterally and axially. Catheter-based ultrasound applicators have been developed that can be inserted into brain tissue through a burr hole under real-time feedback with MRI guidance or through alternative image guidance with separate thermometry sensing. The applicators that we have developed are MR compatible, and thermal dose can be monitored in real time using MR temperature imaging.

\section{Conclusions}

In this study, we characterized our catheter-based ultrasound applicators' ablation patterns in porcine liver tissue, both ex vivo and in vivo, to estimate the lesion size and shape for the respective power and exposure duration. The in vivo results showed direct dependence of the thermal therapy with blood perfusion. Data from PD rats demonstrates the feasibility of treating brain tissue. Brain tissue absorbs ultrasonic energy differently from other soft tissues such as liver, and thus appropriate power/time/temperature determination for optimal dose is needed.

\section{References}

1. Burdette EC, Lichtenstiger C, Rund L, Keralapura M, Gossett C, Stahlhut R, et al: Ultrasound therapy applicators for controlled thermal modification of tissue. Proc SPIE 7901:79010W, 2011

2. Diederich C, Burdette E: Transurethral ultrasound array for prostate thermal therapy: initial studies. IEEE Trans Ultrason Ferroelectr Freq Control 43:1011-1022, 1996

3. Elias WJ, Huss D, Voss T, Loomba J, Khaled M, Zadicario E, et al: A pilot study of focused ultrasound thalamotomy for essential tremor. N Engl J Med 369:640-648, 2013

4. Findley L, Eichhorn T, Janca A, Kazenwadel J, Baker M, Currie-Gnjesda D, et al: Factors impacting on quality of life in Parkinson's disease: results from an international survey. Mov Disord 17:60-67, 2002

5. Gee LE, Chen N, Ramirez-Zamora A, Shin DS, Pilitsis JG: The effects of subthalamic deep brain stimulation on mechanical and thermal thresholds in 6OHDA-lesioned rats. Eur J Neurosci 42:2061-2069, 2015

6. Ghoshal G, Heffter T, Williams E, Bromfield C, Salgaonkar $\mathrm{V}$, Rund L, et al: In situ treatment of liver using catheter based therapeutic ultrasound with combined imaging and GPS tracking. Proc SPIE 8584:85840T

7. Jankovic J, Cardoso F, Grossman RG, Hamilton WJ: Outcome after stereotactic thalamotomy for parkinsonian, essential, and other types of tremor. Neurosurgery 37:680-687, 1995

8. Kangasniemi M, Diederich CJ, Price RE, Stafford RJ, Schomer DF, Olsson LE, et al: Multiplanar MR temperaturesensitive imaging of cerebral thermal treatment using interstitial ultrasound applicators in a canine model. J Magn Reson Imaging 16:522-531, 2002

9. Kemmerer JP, Ghoshal G, Oelze ML, Rupert N, Samimi K: Quantitative imaging of temperature elevations in tissuesdue to thermal therapies, in 2014 IEEE International Ultrasonics Symposium. Piscataway, NJ: IEEE, 2014, pp 2153-2156

10. Kinsey AM, Diederich CJ, Tyreus PD, Nau WH, Rieke V, Pauly KB: Multisectored interstitial ultrasound applicators for dynamic angular control of thermal therapy. Med Phys 33:1352-1363, 2006

11. Lipsman N, Schwartz ML, Huang Y, Lee L, Sankar T, Chapman M, et al: MR-guided focused ultrasound thalamotomy for essential tremor: a proof-of-concept study. Lancet Neurol 12:462-468, 2013

12. Nau WH, Diederich CJ, Burdette EC: Evaluation of multielement catheter-cooled interstitial ultrasound applicators for high-temperature thermal therapy. Med Phys 28:1525-1534, 2001

13. Nau WH, Diederich CJ, Ross AB, Butts K, Rieke V, Bouley DM, et al: MRI-guided interstitial ultrasound thermal therapy of the prostate: a feasibility study in the canine model. Med Phys 32:733-743, 2005

14. Pahwa R, Lyons KE, Wilkinson SB, Simpson RK Jr, Ondo 
WG, Tarsy D, et al: Long-term evaluation of deep brain stimulation of the thalamus. J Neurosurg 104:506-512, 2006

15. Pilitsis JG, Metman LV, Toleikis JR, Hughes LE, Sani SB, Bakay RA: Factors involved in long-term efficacy of deep brain stimulation of the thalamus for essential tremor. J Neurosurg 109:640-646, 2008

16. Scott SJ, Prakash P, Salgaonkar V, Jones PD, Cam RN, Han $M$, et al: Interstitial ultrasound ablation of tumors within or adjacent to bone: contributions of preferential heating at the bone surface. Proc SPIE 8584:85840Z, 2013

17. Sneed PK, Stauffer PR, McDermott MW, Diederich CJ, Lamborn KR, Prados MD, et al: Survival benefit of hyperthermia in a prospective randomized trial of brachytherapy boost \pm hyperthermia for glioblastoma multiforme. Int J Radiot Oncol Biol Phys 40:287-295, 1998

18. Ungerstedt U: 6-hydroxy-dopamine induced degeneration of central monoamine neurons. Eur J Pharmacol 5:107-110, 1968

\section{Disclosures}

Drs. Williams, Ghoshal, Heffter, and Burdette are employees of Acoustic MedSystems. Dr. Pilitsis reports a consultant relation- ship with Boston Scientific, Medtronic, and Abbott (St. Jude Medical); receipt of clinical or research support for the study described from Boston Scientific, Jazz Pharmaceuticals, Nevro, NIH, GE Global Research, Medtronic, and Abbott (St. Jude Medical); receipt of grants from Jazz Pharmaceutical, Nevro, NIH, GE, Boston Scientific, Medtronic, and Abbott (St. Jude Medical); receipt of medical advisor stock equity from Centauri and Karuna; and membership in the speakers' bureaus of Boston Scientific and Jazz Pharmaceutical. Mr. Fischer is a consultant for Centauri.

\section{Author Contributions}

Conception and design: all authors. Acquisition of data: Ghoshal. Analysis and interpretation of data: Ghoshal. Drafting the article: Pilitsis, Ghoshal. Critically revising the article: Pilitsis, Ghoshal. Reviewed submitted version of manuscript: all authors. Approved the final version of the manuscript on behalf of all authors: Pilitsis. Administrative/technical/material support: Pilitsis. Study supervision: Pilitsis.

\section{Correspondence}

Julie G. Pilitsis: Albany Medical College, Albany, NY. pilitsj@ mail.amc.edu. 